\title{
Wnt5a Induces Simultaneous Cortical Axon Outgrowth and Repulsive Axon Guidance through Distinct Signaling Mechanisms
}

\author{
Li Li, ${ }^{1}$ B. Ian Hutchins, ${ }^{1}$ and Katherine Kalil ${ }^{1,2}$ \\ ${ }^{1}$ Neuroscience Training Program and ${ }^{2}$ Department of Anatomy, University of Wisconsin-Madison, Madison, Wisconsin 53706
}

\begin{abstract}
Wnts are morphogens that also function as axon guidance molecules. In vivo Wnt5a gradients via Ryk receptors were found to repel cortical axons into developing callosal and corticospinal pathways. Here, using dissociated cortical cultures, we found that bath-applied Wnt5a increased axon outgrowth. In turning assays, Wnt5a gradients simultaneously increased axon outgrowth and induced repulsive turning, a potential mechanism for propelling cortical axons in vivo. We found that axon outgrowth is mediated by Ryk, whereas axon repulsion requires both Ryk and Frizzled receptors. Both receptors mediate Wnt-evoked fluctuations in intracellular calcium, which is required for increased axon outgrowth and repulsion by Wnt5a. However, whereas increased axon outgrowth involves calcium release from stores through IP3 receptors as well as calcium influx through TRP channels, axon repulsion is mediated by TRP channels without involvement of IP3 receptors. These results reveal distinct signaling mechanisms underlying Wnt5a-induced axon outgrowth and repulsive guidance.
\end{abstract}

\section{Introduction}

In the developing nervous system, growth cones guide axons to appropriate neuronal targets by responding to environmental guidance cues (Dickson, 2002). Wnt proteins are highly conserved secreted morphogens that play important roles during embryogenesis (Ciani and Salinas, 2005). Recent studies have shown that Wnts also function as axon guidance molecules (Bovolenta et al., 2006; Salinas and Zou, 2008). Wnt5 was first identified in Drosophila as a repulsive axon guidance cue for commissural axons (Yoshikawa et al., 2003). In mammals, Wnt5a acts as a repulsive guidance cue for cortical axons in vivo extending through the corpus callosum (Keeble et al., 2006) and the corticospinal tract (Liu et al., 2005). A gradient of Wnt5a was thought to repel cortical axons through the corticospinal tract (Zou and Lyuksyutova, 2007). However, since repulsive guidance cues have not been shown to increase rates of axon outgrowth (Nishiyama et al., 2008), it was not clear how a repulsive guidance cue could promote axon outgrowth of descending cortical axons into the spinal cord.

Wnts signal through the classical Frizzled receptors $(\mathrm{Fz})$ and the atypical tyrosine kinase receptor, Ryk (Logan and Nusse, 2004). Fz receptors appear to mediate growth cone attraction by Wnts in ascending spinal commissural axons (Wnt4) (Lyuksyutova et al., 2003) and in dorsal retinal ganglion cells (Wnt3a)

Received Jan. 13, 2009; revised March 31, 2009; accepted April 2, 2009

This work was supported by National Institutes of Health Grant NS14422 and the Whitehall Foundation (to K.K.), a Herman I. Shapiro Fellowship to L.L., and National Research Service Award GM801642 to B.I.H. We thank Dr. Yimin Zou (University of California, San Diego) for providing the anti-Ryk antibody and Dr. Erik Dent for a critical reading of this manuscript.

Correspondence should be addressed to Dr. Katherine Kalil, Department of Anatomy, University of WisconsinMadison, 1300 University Avenue, Madison, WI 53706. E-mail: kakali@@facstaff.wisc.edu.

D01:10.1523/JNEUROSCI.0183-09.2009

Copyright $\odot 2009$ Society for Neuroscience $\quad$ 0270-6474/09/295873-11\$15.00/0
(Schmitt et al., 2006). Ryk, however, mediates growth cone repulsion in Drosophila commissural axons (Wnt5) (Yoshikawa et al., 2003), mouse corticospinal (Liu et al., 2005) and callosal (Keeble et al., 2006) axons (Wnt5a), and ventral retinal ganglion axons (Wnt3a) (Schmitt et al., 2006). Wnts, by binding to Fz receptors, act through the canonical, planar cell polarity, and Wnt/calcium pathways (Ciani and Salinas, 2005). However, Wnt/Fz signaling pathways have not been extensively studied in the context of axon guidance, and the downstream components of Ryk signaling are unknown.

Here, to identify signaling pathways mediating the responses of cortical axons to Wnt5a, we used dissociated cultures of $2 \mathrm{~d}$ hamster sensorimotor cortex, when cortical axons begin to enter the corticospinal tract (Reh and Kalil, 1981) and corpus callosum (Norris and Kalil, 1992) and would encounter Wnt5a gradients. Surprisingly, bath application of Wnt5a increased cortical axon outgrowth, but when Wnt5a was applied as a gradient, cortical axons were not only repelled from Wnt5a but simultaneously increased their rates of axon outgrowth. This suggests a mechanism whereby Wnt5a, by growth cone repulsion, could propel cortical axons through their efferent pathways. Our investigation of Wnt5a signaling shows that axon outgrowth is dependent on Ryk receptors, whereas growth cone repulsion requires both Ryk and Fz. Both receptors mediate Wnt-evoked calcium transients, which are necessary for Wnt5a-induced axon outgrowth and repulsion. However axon outgrowth involves calcium release through IP3 receptors as well as calcium influx through TRP channels, whereas growth cone repulsion is mediated by TRP channels but not IP3 receptors. Together, these results suggest that Wnt5a, acting through distinct Wnt receptors and calcium signaling pathways, can simultaneously increase cortical axon outgrowth and induce repulsive axon guidance. 


\section{Materials and Methods}

Experimental reagents. The following reagents were used: culture media and electrophoresis reagents (Invitrogen), bovine serum albumin (BSA, Sigma), recombinant protein Wnt5a (R\&D Systems), secreted Frizzledrelated protein (sFRP-2, R\&D Systems), anti-mouse Frizzled-2 antibody (R\&D Systems), rabbit-anti-Ryk function-blocking antibody (a gift from Dr. Yimin Zou, University of California, San Diego, La Jolla, CA), mouse-anti- $\beta$ III tubulin antibody (Millipore), ECL Plus (GE Healthcare), 2-aminoethoxydiphenyl borate (2-APB, EMD Biosciences), SKF96365 (Alexis Biochemicals), BAPTA-AM (Invitrogen), U-37122 (Sigma), m-3M3FBS (EMD Biosciences), autocamtide-2-related inhibitory peptide II (AIPII, EMD Biosciences), KN62 (EMD Biosciences), 1-oleoyl-2-acetyl-sn-glycerol (OAG, Sigma-Aldrich), and IgG (Santa Cruz Biotechnology).

Dissociated cell culture and bath application of reagents. Cultures were prepared from sensorimotor cortex obtained from postnatal $0-3 \mathrm{~d}(\mathrm{P} 0-$ P3) golden Syrian hamsters (Mesocricetus auratus) as described previously (Dent and Kalil, 2003). Dissociated primary neurons were plated onto coverslips coated with $0.5 \mathrm{mg} / \mathrm{ml}$ poly-D-lysine (Sigma) and 20 $\mu \mathrm{g} / \mathrm{ml}$ laminin (Sigma/Invitrogen) at a density of $2000-7000 \mathrm{cells} / \mathrm{cm}^{2}$ (500,000 cells $/ \mathrm{cm}^{2}$ for Western blotting) and were incubated in $5 \% \mathrm{CO}_{2}$ and $9 \% \mathrm{O}_{2}$ at $37^{\circ} \mathrm{C}$. In some experiments, etched coverslips (Bellco) were used to locate neurons. For $3 \mathrm{~d}$ experiments, $1 \mathrm{~h}$ after plating, cortical neurons from P2 hamsters were flooded with serum-free medium, to which various reagents were added, and incubated for $72 \mathrm{~h}$. For $1 \mathrm{~h}$ experiments, neurons were obtained from P2 cortex and maintained in culture for $1 \mathrm{~d}$ before the experiments.

Measurements of axon outgrowth and statistical analysis. Measurements of axon outgrowth and branching after bath application of reagents for $3 \mathrm{~d}$ were performed on phase images of fixed neurons, which were acquired with a $20 \times 0.5$ numerical aperture (NA) Plan Fluor objective mounted on a Nikon TE300 Quantum inverted microscope equipped with a Princeton Instruments MicroMax 512 BFT cooled CCD camera or a Photometrics Cascade II: 512. Only neurons with pyramidal morphologies and with a process $>100 \mu \mathrm{m}$ were chosen for analysis. Such neurons were likely to be cortical projection neurons. Axon lengths were measured with the morphometric analysis tools of MetaMorph (Molecular Devices) from the cell body to the distal extent of the central region of the growth cones. An axon was defined as a process that remains parallel to the initial axon segment extending from the cell body. Only processes longer than $20 \mu \mathrm{m}$ were included as branches. Measurements of axon outgrowth in $1 \mathrm{~h}$ time periods were made from images of live cells at the beginning and end of the experiment captured with a $20 \times 0.50 \mathrm{NA}$ Plan Neofluar objective mounted on a Zeiss $35 \mathrm{M}$ inverted microscope. Data were saved in Excel 2000 (Microsoft) and GraphPad Prism (Graph$\mathrm{Pad}$ ) was used to perform statistical analysis and create graphs. In experiments comparing 2 treatments and in turning assays the Mann-Whitney test was used for statistical analysis. For experiments comparing effects of multiple treatments, one-way ANOVA was performed followed by Dunnett's posttest. Measurements are given in mean \pm SEM unless otherwise noted.

Turning assays. Neurons were dissociated from P2 cortex and turning assays were performed in Leibovitz-15 (L15) medium (Invitrogen) on neurons cultured for $1-3 \mathrm{~d}$. Pharmacological reagents were added to the medium $30 \mathrm{~min}$ before the turning assays.

Turning assays were performed on neurons with a straight $20 \mu \mathrm{m}$ segment at the end of the axon. A pipette with a $1 \mu \mathrm{m}$ tip diameter was filled with either BSA $(0.5 \%)$ or Wnt5a $(10 \mu \mathrm{g} / \mathrm{ml})$. The pipette was positioned $100 \mu \mathrm{m}$ away from the axonal growth cone at a $45^{\circ}$ angle from the original direction of axon outgrowth, which was defined by the direction of the $20 \mu \mathrm{m}$ axon segment. Two hertz pulses (3 psi, $20 \mathrm{~ms}$ ) were generated with a pulse generator (Grass Product Group, SD9) and a Picospritzer II (General Valve). Gradients were confirmed by using fluorescent dyes (data not shown). These methods were similar to turning assays performed on Xenopus spinal axons (Lohof et al., 1992; Zheng et al., 1994). Phase images of live cells were acquired with the Nikon microscope at $1 \mathrm{~min}$ intervals to record growth cone behaviors. Outgrowth rates were quantified by tracing trajectories of the part of the axon that grew out during the $1 \mathrm{~h}$ time period. Turning angles were defined as the angles between the original direction of axon outgrowth and a line connecting the original position of the growth cone and its final position. To display axon trajectories, traces of individual axons were made from the final image of each axon at the end of the $1 \mathrm{~h}$ turning assay.

Immunocytochemistry and Western blotting. Immunocytochemistry was performed as described previously (Dent and Kalil, 2001). Neurons were not permeabilized to stain only membrane proteins. Briefly, neurons from $\mathrm{P} 0-\mathrm{P} 3$ cortex were cultured for $1-3 \mathrm{~d}$, fixed with $4 \%$ paraformaldehyde (Electron Microscopy Sciences) in Krebs' buffer, pH 7.4, blocked by using $5 \%$ normal donkey serum (Millipore Bioscience Research Reagents), incubated with diluted primary antibodies (1:50 rabbit anti-Ryk antibody and 1:10 rat anti-Frizzled 2 antibody) at $4^{\circ} \mathrm{C}$ overnight, and then incubated with diluted secondary antibodies (1:400 Cy2conjugated donkey anti-rabbit IgG, Jackson ImmunoResearch, or 1:400 Cy2-conjugated donkey anti-rat IgG, Jackson ImmunoResearch) at room temperature for $1 \mathrm{~h}$. Fluorescence images were acquired with a $60 \times$ 1.40 NA Plan Apo objective mounted on the Nikon microscope. In Western blotting, RIPA (Thermo Fisher Scientific) supplied with protease inhibitor cocktail tablets (Roche) were used to extract total proteins from P0 sensorimotor cortex dissociated and cultured for 3-4 d. Blots blocked with 3\% milk (Lab Scientific) and 3\% BSA were incubated with rabbit anti-mouse Ryk antibody (1:500) and goat anti-rabbit-HRP (1:10,000, Jackson ImmunoResearch) to stain Ryk receptors. Antibodies were then stripped by reblot plus mild solution (Millipore Bioscience Research Reagents) and reprobed with mouse anti- $\beta$ III tubulin antibody (1:500) and goat anti-mouse-HRP (1:10,000, Jackson ImmunoResearch) to stain the loading control, $\beta$ III tubulin.

RNAi and electroporation. ON-TARGETplus Non-Targeting Pool siRNA and ON-TARGETplus SMARTpool mouse Ryk siRNA, sequenced as CAAAGUCCAAGGUUGAAUAUU, CAAAUUAGUAGAAGCCAAUUU, AAUGAGUUCUCUAGUGCUAUU, and GCAGUGAUAUUUCUUGUAGUU, were purchased from Dharmacon, and electroporation was performed with a Nucleofector (Amaxa) and Mouse Neurons Kit (Amaxa) according to the recommended protocol. For each transfection, neurons obtained from 1-2 P0 hamsters were electroporated with 100 pmol Ryk siRNA and 56 pmol pMax GFP (Amaxa). Expression of GFP could be detected $2 \mathrm{~d}$ after transfection, and experiments were performed on transfected neurons cultured for 2-3 d. Cotransfection efficiency was tested by transfecting GFP and RFP at the same time. Over $60 \%$ of neurons cultured for $2 \mathrm{~d}$ expressed GFP while cotransfection efficiency was $\sim 90 \%$. A second pool of Ryk siRNAs, sequenced as CCAUGGUGGUAUUGCCAUA, CCAUAUGGCUAUUCAGAUU, and CAAGGCUGGCUUAAGUAUA, was purchased from Santa Cruz Biotechnology, and the same protocol was used for the electroporation.

Measurements of calcium activity. Calcium imaging and analysis were performed as previously described (Hutchins and Kalil, 2008). Briefly, $18-72 \mathrm{~h}$ after plating cultured neurons were loaded with $4 \mu \mathrm{M}$ Fluo- $4 \mathrm{AM}$ (Invitrogen) for $30 \mathrm{~min}$. DIC and fluorescence microscopy were performed with a $60 \times 1.40$ NA Plan Apo objective mounted on the Nikon microscope. Fluorescence time-lapse images were taken every 5-10 s. Cultures were maintained at $37^{\circ} \mathrm{C}$ with an incubator blower. Calcium activity was measured as the average fluorescence pixel intensity $(F)$ in an axon region divided by the baseline fluorescence in that region $\left(F_{0}\right)$. Background fluorescence was measured frame by frame in an empty region of the viewing field and was subtracted from measurements of fluorescence intensity. To minimize the effects of any morphological changes that could affect fluorescence measurements through changes in volume, the baseline $\left(F_{0}\right)$ was calculated as a shifting average of the fluorescence intensity over a $5 \mathrm{~min}$ window. Calcium transients were defined as fluorescence signals $\left(F / F_{0}\right)$ that exceed $150 \%$ of the baseline fluorescence, which were confirmed by frame-by-frame analysis of the time-lapse images. To ensure that random fluctuations were not counted as calcium transients, we included a requirement that the peak amplitude of transients be at least 2 SDs from baseline. After imaging, the frequency of calcium transients was measured and analyzed with computer algorithms in Microsoft Excel. For ratiometric experiments, neurons were loaded with $4 \mu \mathrm{M}$ Fluo-4 AM and $250 \mathrm{~nm}$ volumetric indicator Calcein Red/Orange (Invitrogen) for $30 \mathrm{~min}$. Fluorescence images of Calcein 
Red/Orange were acquired immediately after each frame of Fluo-4 fluorescence. Ratiometric measurements $(R)$ were obtained by dividing the Fluo-4 fluorescence value by the fluorescence value of Calcein Red/Orange. Frame-by-frame background subtraction was performed for each indicator as described above. Calcium signals $\left(R / R_{0}\right)$ were then measured as the percentage change from a shifting average baseline $\left(R_{0}\right)$ of the ratiometric measurements as described above for nonratiometric measurements.

\section{Results}

\section{Wnt5a increases outgrowth rates of cortical axons}

To investigate in vitro the effects of Wnt5a on the growth and guidance of cortical axons, we first bath applied purified Wnt5a to dissociated cultures of hamster sensorimotor cortex at $2 \mathrm{~d}$ postnatal. We could not specifically identify projection neurons, but we chose for study large pyramidal neurons that are likely to be long distance projection neurons. After exposing the cultures to Wnt5a at a concentration of $400 \mathrm{ng} / \mathrm{ml}$ for $3 \mathrm{~d}$, we measured axonal and branch length in comparison with control axons exposed to BSA. We found that the average length of axons exposed to Wnt5a $(n=87)$ was double that of control axons $(n=83)$ (Fig. 1C). Control axons averaged $327 \mu \mathrm{m}$ with several branches after $3 \mathrm{~d}$ in culture, whereas cortical axons exposed to Wnt5a averaged $577 \mu \mathrm{m}$ and could reach lengths of $>1200 \mu \mathrm{m}$ (Fig. $1 A-D$ ). Wnt5a did not increase the number of axon branches (data not shown), but branch length was also doubled (Fig. 1C). Increasing concentrations of Wnt5a had increasing effects on axon length (Fig. $1 E$ ). When we examined axon outgrowth after shorter exposures to Wnt5a, we found that within $1 \mathrm{~h}$ the rate of axon outgrowth was 4 times that of control axons (1.7 vs $7.7 \mu \mathrm{m} / \mathrm{h}$ ) (Fig. $1 F-I)$. These results show that $\mathrm{Wnt} 5 \mathrm{a}$ is a potent and rapid promoter of cortical axon outgrowth.

\section{Wnt5a simultaneously induces axon outgrowth and repulsive axon guidance}

In explant cocultures Wnt5a secreted from spinal cord explants or COS cells had repulsive effects on the growth of axons from explants of neonatal mouse sensorimotor cortex (Liu et al., 2005). To determine whether a point source of Wnt5a would exert a repulsive guidance effect on cortical axons, we used a turning assay in which cortical axons were exposed to a gradient of Wnt5a delivered from a pipette attached to a pressure ejection system. The establishment of gradients with these techniques was confirmed with fluorescent dyes (data not shown). As shown in Figure $2 A$ and supplemental Movie 1 (available at www.jneurosci.org as supplemental material), control axons exposed to BSA pulsed from a pipette positioned at $45^{\circ}$ angle $100 \mu \mathrm{m}$ from the axon tip grew at an average rate of $14 \mu \mathrm{m} / \mathrm{h}$ (Fig. $2 D)$. This rate of outgrowth was greater than that measured in bath application, since in these experiments we only included axons that extended at $>5 \mu \mathrm{m} / \mathrm{h}$. When the final trajectories of these axons $(n=20)$ were traced after $1 \mathrm{~h}$ the axons showed no preference in extension toward or away from the source of the BSA (Fig. $2 \mathrm{~A}$, right; supplemental Movie 1, available at www.jneurosci.org as supplemental material). In contrast, when Wnt5a was pulsed onto cortical axons they consistently turned away from the source of Wnt5a. As shown in the time-lapse images, growth cones could reorient sharply away from the pipette in as little as $20 \mathrm{~min}$ (Fig. $2 \mathrm{~B}$; supplemental Movie 2, available at www.jneurosci.org as supplemental material) while remaining highly motile without any obvious collapse on the side facing the pipette. The cumulative distribution plotting the turning angles of all the axons plotted in Figure $2 D$ shows that axons exposed to Wnt5a have primarily negative turning angles in comparison with those exposed to BSA (Fig. 2C). Repulsive turning angles for axons exposed to Wnt5a averaged $-23^{\circ}$ (Fig. 2D). Surprisingly, axons responding to $\mathrm{Wnt5a}$ with repulsive turning also increased their average rates of outgrowth to $20 \mu \mathrm{m} / \mathrm{h}$ (Fig. $2 D$ ), an increase of almost $50 \%$ over controls.

\section{Wnt5a induces axon growth and guidance differentially through Ryk and Frizzled receptors}

Wnts exert their effects through both Ryk and Frizzled receptors (Bovolenta et al., 2006). The effects of Wnt5a on the repulsive guidance of cortical axons in vivo are mediated by Ryk, which is expressed in mouse cortex (Liu et al., 2005; Keeble et al., 2006). In dissociated cultures, we sought to determine which receptors are involved in the Wnt5a-induced growth and guidance of cortical axons. We first confirmed that both receptor types are present on cortical neurons at the relevant postnatal ages. As shown in Figure $3 A$, immunocytochemistry with antibodies to Ryk stained the entire cortical neuron, and Ryk receptors were present on cortical neurons from P0 to P3, which were cultured for 2-3 d before immunostaining. The presence of Ryk receptors was confirmed with Western blotting (Fig. 3B). Evidence from in situ hybridization has shown that seven different Fz receptors, including Fz2, are present in early postnatal mouse cerebral cortex (Shimogori et al., 2004). We were interested in the role of calcium signaling in effects of Wnt5a on cortical neurons. We therefore chose Fz2 for immunostaining since previous studies (Slusarski et al., 1997; Veeman et al., 2003a; Kohn and Moon, 2005) showed that Wnt5a-induced calcium signaling in zebrafish embryos is mediated by Fz2 receptors. We found that cortical neurons expressed Fz2 at ages $\mathrm{P} 0-\mathrm{P} 3$ (Fig. 3A), when cortical axons are projecting into efferent corticospinal and callosal pathways (Reh and Kalil, 1981; Norris and Kalil, 1992). Double staining showed that single neurons could express both Ryk and Fz2 receptors (Fig. 3A), which were distributed over all the processes of pyramidal neurons. A quantitative analysis showed that $87 \pm 7 \%$ of neurons (P2, 2-3 DIV) expressed Ryk receptors, $98 \pm 1 \%$ expressed Fz2 receptors and $87 \pm$ $7 \%$ expressed both receptors (total $n=136$ in three independent experiments). Thus, for the relevant sensorimotor cortical neurons almost all expressed both Ryk and Fz receptors and would be capable of responding to Wnt/Ryk and Wnt/Fz signaling.

To determine whether Ryk receptors are involved in Wnt5amediated axon outgrowth, we applied function-blocking antibodies to Ryk $(10 \mu \mathrm{g} / \mathrm{ml})$ (Liu et al., 2005). As shown in Figure 3C-G, blocking Ryk receptors prevented the increased axon outgrowth by bath-applied Wnt5a. As a control for the Ryk antibody, we applied $10 \mu \mathrm{g} / \mathrm{ml}$ rabbit IgG, an isotype-matched, nonspecific primary antibody. As shown in supplemental Figure $3 A$ (available at www.jneurosci.org as supplemental material), this IgG did not block Wnt5apromoted axon outgrowth. As a second approach we used Ryk siRNA knockdown, which effectively reduced Ryk levels by $20-60 \%$ (Fig. 3B). Previous studies of the expression of Ryk receptors in embryonic mouse brain (Schmitt et al., 2006), in transfected HEK cells (Trivier and Ganesan, 2002) and in human breast carcinoma cells (Halford et al., 1999) have revealed several different forms of the full-length Ryk protein ranging from molecular masses of 70-100 $\mathrm{kDa}$, consistent with our immunoblot that identified a $100 \mathrm{kDa}$ fulllength Ryk. Our results suggest proteolytic cleavage of the Ryk protein into 50 and $60 \mathrm{kDa}$ fragments, which has been demonstrated biochemically in mouse kidney lysate (Halford et al., 2000) and recently shown in developing cortical neurons (Lyu et al., 2008). Under both reducing and nonreducing conditions, we obtained similar results for the molecular weights of Ryk. The Ryk siRNA consisted of a pool of four different Ryk siRNAs, a strategy to reduce off-target effects (Dharmacon, see Materials and Methods). In addition, we used a control siRNA to exclude the possibility of general depression of protein expression. As shown in the Western blots in Figure 3B, 


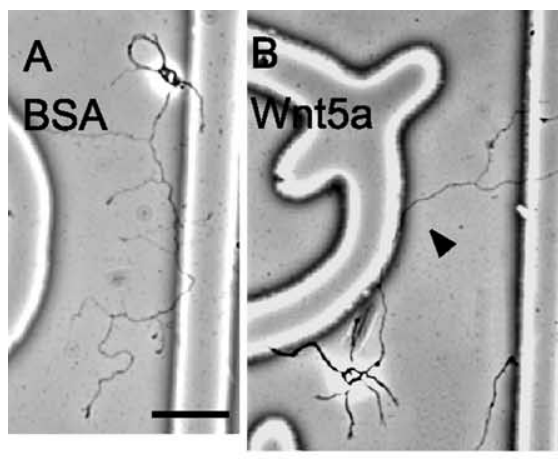

C

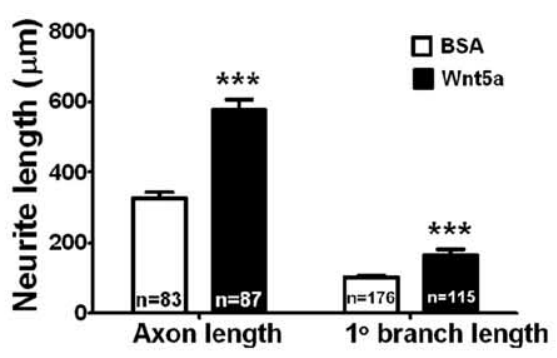

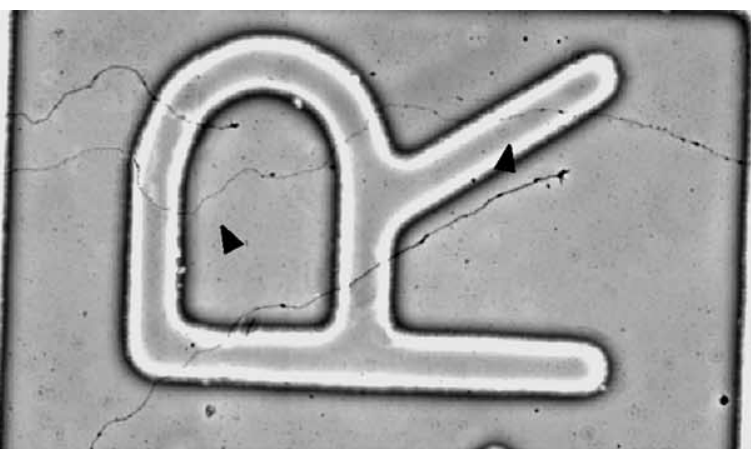

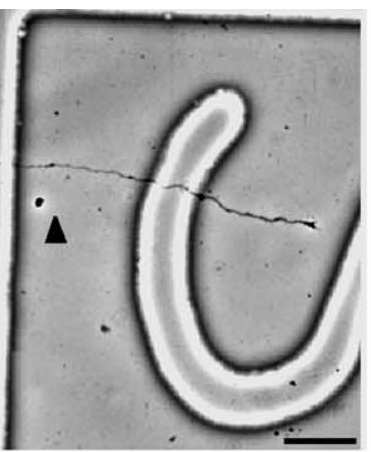

E

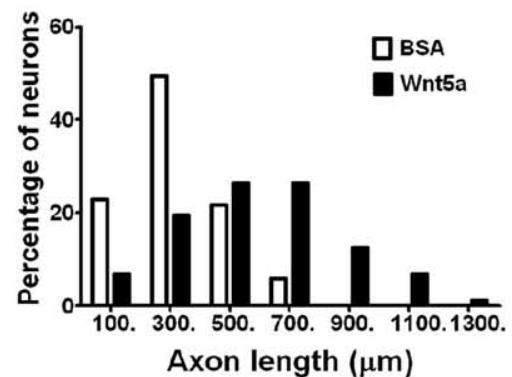

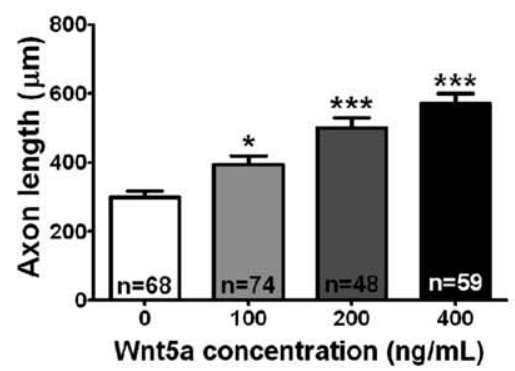

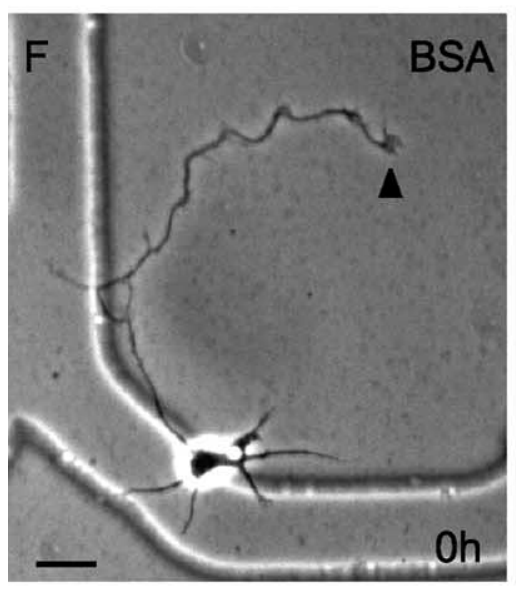

$\mathrm{H}$

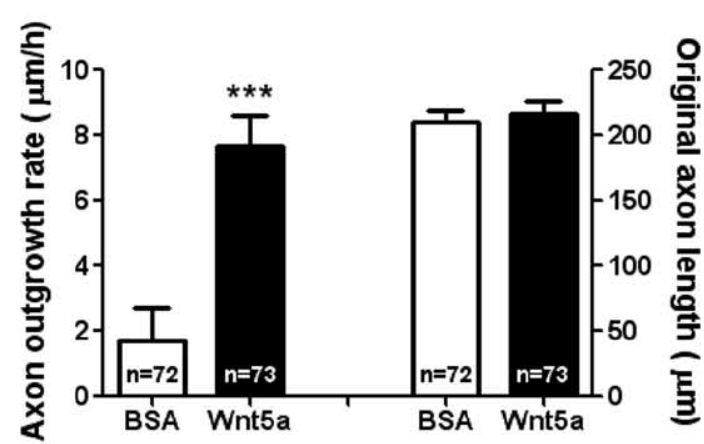

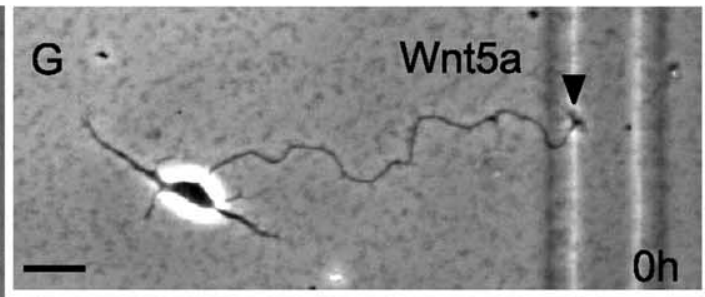

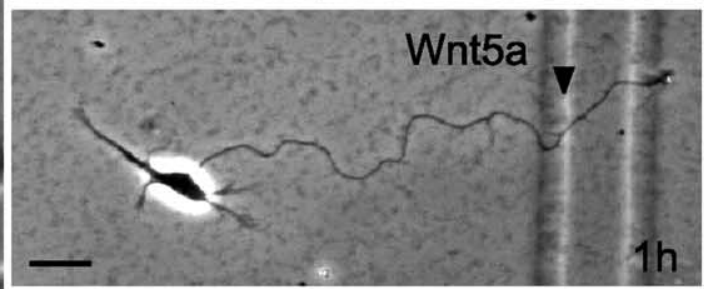

I

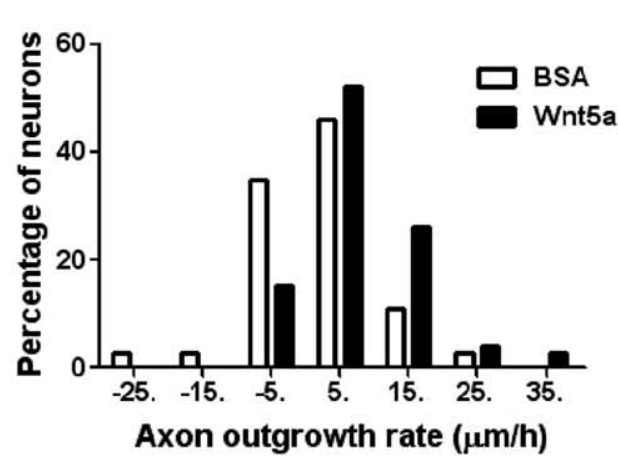

Figure 1. Wnt5a applied in the bath increases rates of axon outgrowth. $\boldsymbol{A}$, Axon outgrowth is $302 \mu \mathrm{m}$ in control medium with BSA over $3 \mathrm{~d}$ in culture. $\boldsymbol{B}$, Axon outgrowth is $1085 \mu \mathrm{m}$ in the presence of $400 \mathrm{ng} / \mathrm{ml}$ Wnt5a. Note that only a single branch is present, which also elongates more rapidly than in control cultures, but Wnt5a does not increase numbers of branches. Arrowheads indicate the primary axon. Scale bar, $50 \mu \mathrm{m}$. C, Bar graphs showing that treatment ( $3 \mathrm{~d}$ ) with Wnt5a ( $400 \mathrm{ng} / \mathrm{ml})$ increases axon and branch length. Numbers show axons measured in a single experiment, and all experiments were repeated three times. $\boldsymbol{D}$, Histograms showing that Wnt5a increases the percentage of neurons with long axons. $\boldsymbol{E}$, Axon lengths measured in increasing concentrations of Wnt5a. $\boldsymbol{F}, \mathbf{G}$, Examples of axon outgrowth in $1 \mathrm{~h}$ in BSA control cultures and in Wnt5a-treated cultures. Arrowheads indicate the initial position of the growth cone. $\boldsymbol{H}$, Comparisons of axon outgrowth in neurons treated with BSA and Wnt5a (left), showing a fourfold increase in rate of axon outgrowth with Wnt5a treatment for $1 \mathrm{~h}$. Comparison of original axon lengths at the beginning of the experiments (right) showing no difference. Numbers represent the total from three experiments combined. $I$, Histograms showing that Wnt5a increases the percentage of axons with fast outgrowth rates. Scale bar, $20 \mu \mathrm{m}$. In all histograms, ${ }^{*} p<0.05$ and ${ }^{* * *} p<0.001$. 

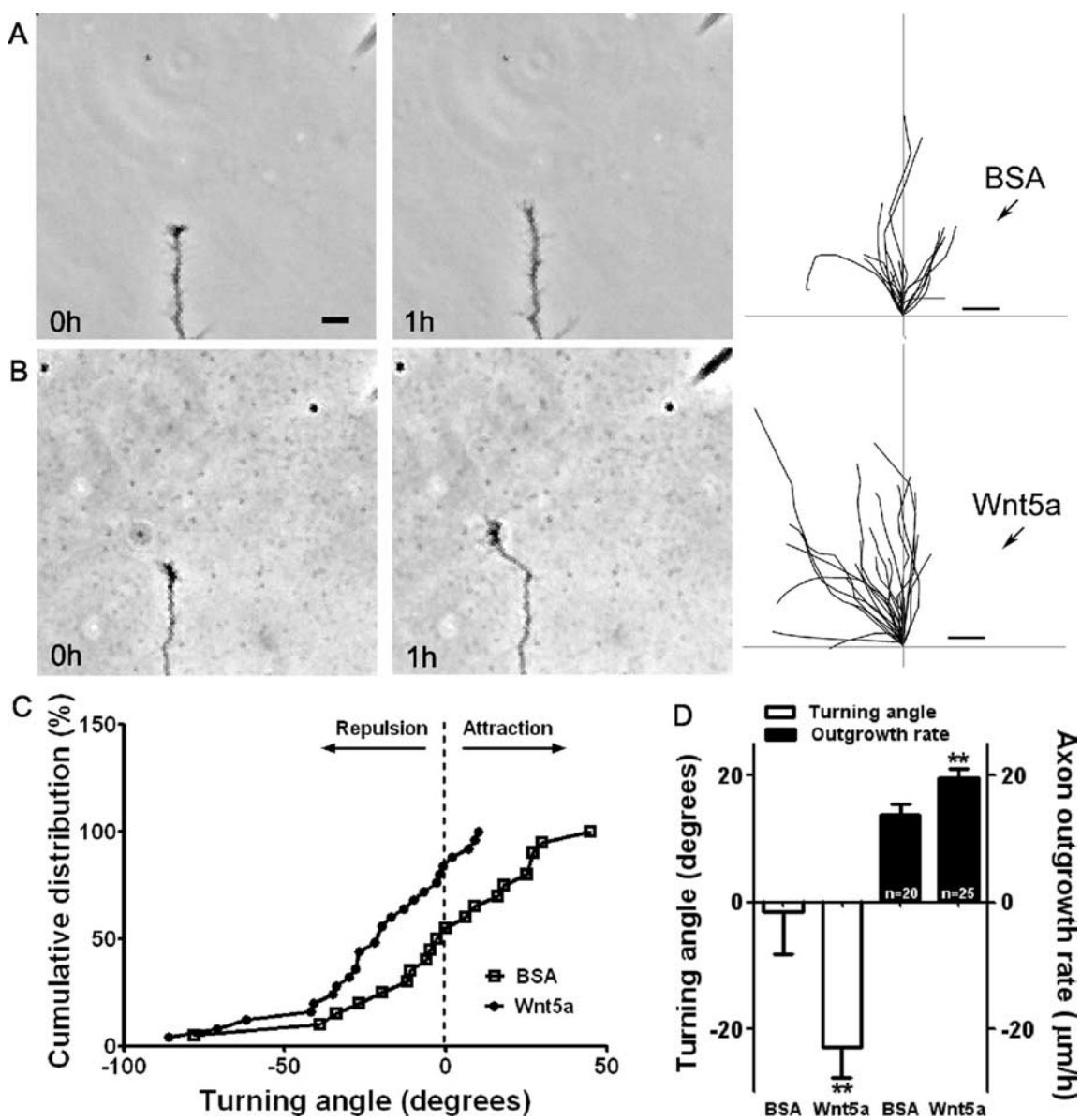

Figure 2. Wnt5a applied as a gradient simultaneously increases axon outgrowth and induces repulsive axon turning. $\boldsymbol{A}, \boldsymbol{B}$, Time-lapse images of a cortical axon and its growth cone at the beginning and end of a $1 \mathrm{~h}$ exposure to a point source of BSA $(\boldsymbol{A})$ or $10 \mu \mathrm{g} / \mathrm{ml}$ Wnt5a $(\boldsymbol{B})$ emitted by the pipette at right. The axon extends straight in the BSA gradient but is repelled by the Wnt5a gradient. Right, Tracings of trajectories of individual axons extending during $1 \mathrm{~h}$ exposure to BSA ( $n=20$ axons) or Wnt5a gradients ( $n=25$ axons) showing random turning in the presence of BSA and repulsive turning in response to Wnt5a. Note that axons repelled by Wnt5a are also significantly longer. $C$, Cumulative distribution of turning angles of axons showing random turning in BSA and repulsive turning in Wnt5a gradients. D, Bar graphs comparing average turning angles and average axon outgrowth rates in BSA and Wnt5a. In all experiments, neurons were obtained from P2 pups and cultured for 2-3 d before the turning assays. Scale bar, $5 \mu \mathrm{m}$. In all histograms, ${ }^{* *} p<0.01$.

Ryk siRNA knockdown reduced levels of Ryk in comparison with mock-transfected and control siRNA-transfected neurons, respectively. Compared with mock transfection, control siRNA does not reduce protein expression (data not shown). Transfection of cortical neurons with Ryk siRNA prevented increased axon outgrowth in the presence of Wnt5a (Fig. 3H-L), demonstrating the requirement of Ryk in mediating Wnt5a-promoted axon outgrowth. Transfection with a new unrelated pool of Ryk siRNAs gave similar results (supplemental Fig. 3B, available at www.jneurosci.org as supplemental material).

To determine whether the effects of Wnt5a on cortical axon outgrowth are also mediated by Fz receptors, we applied secreted Frizzled-related protein 2 (sFRP2), which is known to block Wnt/ Frizzled interaction (Bafico et al., 1999; Rodriguez et al., 2005). This treatment in the presence of Wnt5a did not prevent increased axon outgrowth (Fig. 3M-Q), and axon lengths were similar to those in neurons treated with Wnt5a alone. These results show that Wnt5a-evoked increases in axon outgrowth are mediated by Ryk but not Fz receptors.

To determine which receptors mediate the effects of Wnt5a on repulsive cortical axon guidance, we first bath applied sFRP2 to P2

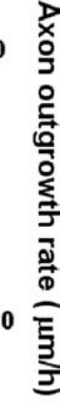

cortical neurons. In the Wnt5a turning assay, axons were unable to respond to the Wnt5a gradient with repulsive turning and grew out in random trajectories (Fig. $4 A, C$ ). Importantly, although axons showed no turning preferences, they increased their rates of axon outgrowth during the $1 \mathrm{~h} \mathrm{ex}$ periments (Fig. 4C). Similarly as shown in Figure $3 M-Q$, when sFRP2 was applied to cultures exposed to bath-applied Wnt5a, there was no decrease in Wnt5a-promoted axon outgrowth. Thus, in both short-term (1 h) and long-term ( $3 \mathrm{~d}$ ) experiments, Wnt5apromoted axon outgrowth does not require $\mathrm{Fz}$ receptors. To knock down Ryk with siRNA, it was necessary to use $\mathrm{P} 0$ neurons to obtain high transfection efficiency. Neurons were cultured for 2-3 d after transfection before the turning assays were carried out. In the turning assays, axons were unable to respond to Wnt5a with repulsive growth cone turning (Fig. 4B, C). Moreover, knockdown of Ryk with siRNA also prevented the ability of Wnt5a to increase axon outgrowth in the $1 \mathrm{~h}$ turning assay (Fig. 4C). This result is similar to those from $3 \mathrm{~d}$ experiments in which both function-blocking Ryk antibodies and Ryk siRNA knockdown were shown to prevent the outgrowth-promoting effects of bath-applied Wnt5a (Fig. 3C-L). Thus, in both short term $(1 \mathrm{~h})$ and long term $(3 \mathrm{~d})$, Ryk receptors are required for Wnt5apromoted axon outgrowth. Interestingly, although axons of $\mathrm{P} 0$ neurons are repelled by Wnt5a, the turning angles are smaller in comparison with P2 neurons (Fig. 4C). This suggests that neurons at the developmental age (P2), when axons are entering the spinal cord and corpus callosum, may be more responsive to the repulsive guidance effects of Wnt5a than neurons at P0. Together these results show differential roles for Ryk and $\mathrm{Fz}$ receptors. Ryk but not Fz receptors are involved in Wnt5a-evoked increases in cortical axon outgrowth, whereas both Ryk and Fz receptors mediate repulsive cortical axon guidance induced by Wnt5a.

\section{Wnt5a evokes calcium activity through both Ryk and Fz receptors}

Calcium signaling is essential in transducing effects of growth and guidance factors on axonal growth cones (Henley and Poo, 2004; Gomez and Zheng, 2006). Previous studies have shown that $\mathrm{Fz}$ receptors are involved in Wnt/calcium signaling during morphogenic events and in cell motility (Slusarski et al., 1997; Veeman et al., 2003a; Kohn and Moon, 2005). However, the role of Ryk receptors in calcium signaling is unknown and Wnt/calcium signaling has not been studied during axon growth and guidance. To address these questions, we first asked whether Wnt5a can induce calcium transients in cortical neurons. We bath applied Wnt5a and performed calcium imaging with the calcium indicator Fluo-4. Within 10-20 min, Wnt5a evoked calcium transients with an average frequency of $15.6 \pm 2.1$ transients/h in $63 \%$ of neurons that lacked spontaneous activity (37/59 neurons) (Fig. $5 A, B$ ). In the example shown in Figure $5 A$ 

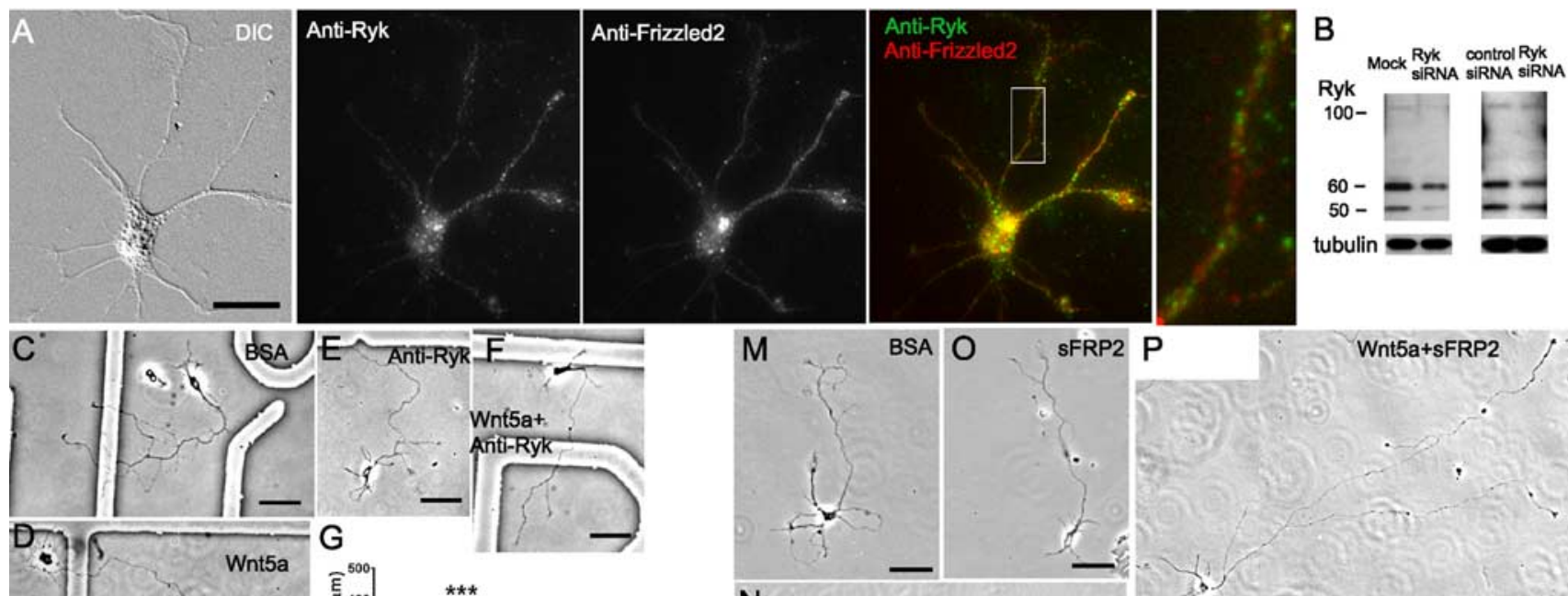

Wnt5a+sFRP2
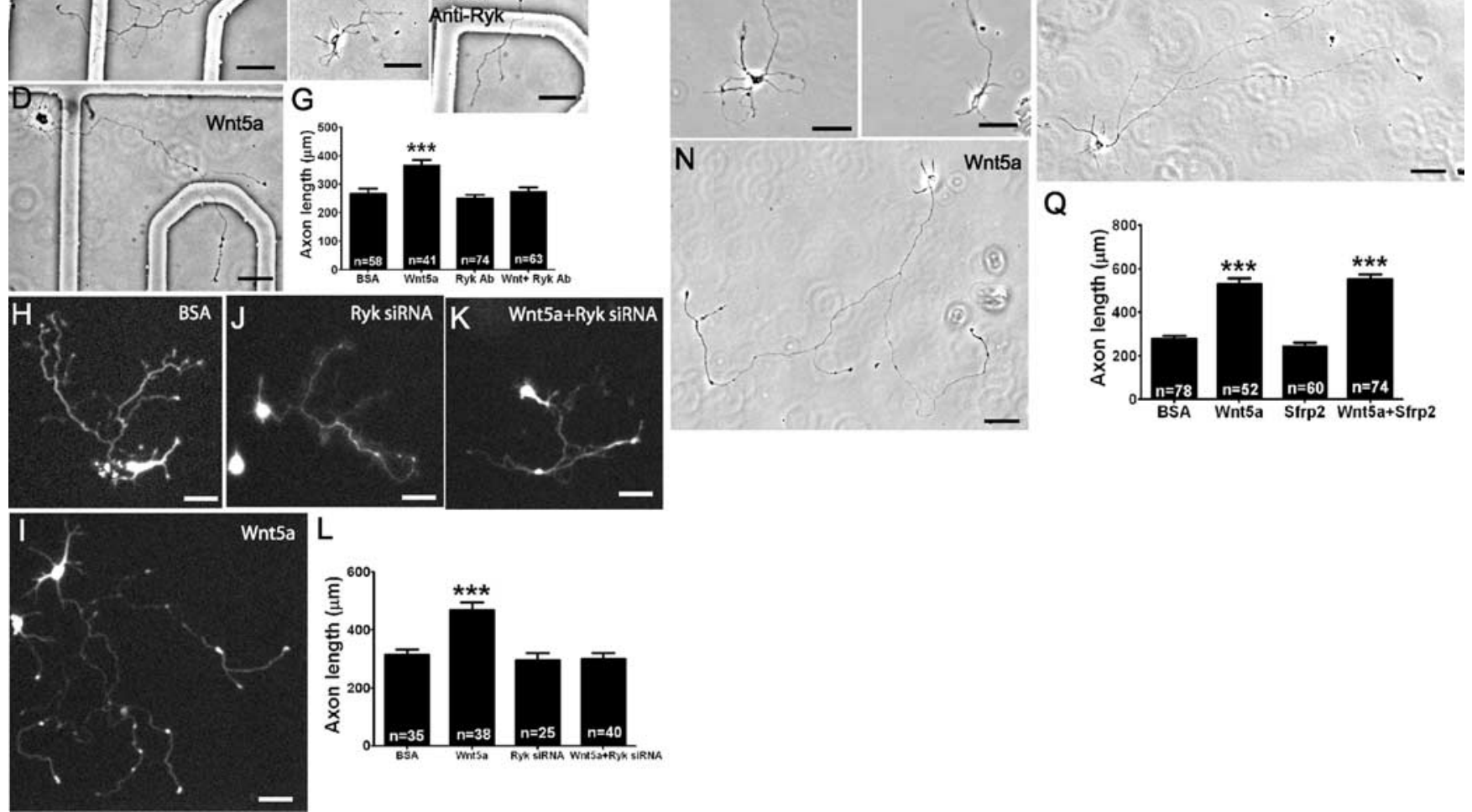

Figure 3. Ryk but not Fz receptors mediate Wnt5a-induced axon outgrowth. Immunocytochemistry with anti-Ryk and anti-Frizzled2 antibodies ( $\boldsymbol{A}$ ) show the presence of Ryk and Fz2 receptors on a pyramidal neuron from $2 \mathrm{~d}$ sensorimotor cortex cultured for 2-3 d before staining (scale bar, $20 \mu \mathrm{m}$ ). Left, A DIC image of the neuron stained for Ryk (green) and Fz2 (red) and the overlay showing both receptors are stained on the same neuron. Inset at right is from the box area at higher power. $B$, Western blot showing at left the presence of Ryk in PO sensorimotor cortical neurons after 3-4 $\mathrm{d}$ in culture compared with neurons from the same tissue that were transfected with $100 \mathrm{pmol}$ of Ryk siRNA. At right, the Western blot shows a decrease in Ryk expression following Ryk siRNA compared with a pool of nontargeting control siRNAs. $(-\boldsymbol{F}$, Phase images of cortical neurons treated with BSA (control), Wnt5a, a function-blocking anti-Ryk antibody (10 $\mu \mathrm{g} / \mathrm{ml})$, or Wnt5a in the presence of anti-Ryk. $\boldsymbol{H}-\boldsymbol{K}$, Fluorescence images of cortical neurons transfected with GFP and treated with BSA (control) or Wnt5a, or transfected with GFP and Ryk siRNA and treated with BSA or Wnt5a. $\boldsymbol{M - P}$, Phase images of cortical neurons treated with BSA, Wnt5a, $200 \mathrm{ng} / \mathrm{ml}$ sFRP2 (which blocks Wnt/Fz interaction), and sFRP2 in the presence of Wnt5a. Scale bar: $\mathbf{C}-\mathbf{Q}, 50 \mu \mathrm{m} . \mathbf{G}, \mathbf{L}$, $\mathbf{Q}$, Bar graphs of axon lengths of cortical neurons in conditions shown in $\mathbf{C}-\boldsymbol{F}, \boldsymbol{H}-\boldsymbol{K}$, and $\boldsymbol{M}-\boldsymbol{P}$, respectively. $n$, Numbers of axons in each condition in one experiment. Experiments were repeated three times. In all histograms, ${ }^{* * *} p<0.001$.

and supplemental Movie 3 (available at www.jneurosci.org as supplemental material), calcium activity was evoked in the growth cone and the adjacent distal axon, but in other cases Wnt5a could evoke global (35\%), localized (49\%), or both (16\%) types of activity in the responsive neurons. Local application of Wnt5a can also induce localized and global calcium activity in cortical growth cones (data not shown). To determine which receptor types were involved in calcium signaling, we bath applied Wnt5a to cortical neurons in which Ryk receptors were knocked down with Ryk siRNA or in which Fz receptors were blocked with sFRP2 (Fig. 5C,D). When calcium activity was measured in these neurons, we found that both receptor types were involved in calcium signaling. In cells treated with Ryk siRNA, calcium activity was evoked by Wnt5a in only $25 \%$ of the neurons $(3 / 12)$ and in $23 \%$ of neurons treated with sFRP2 (3/13) (Fig. 5J).
This substantial reduction in calcium activity in both experiments compared with control neurons shows that Wnt5a can evoke calcium signaling in cortical neurons equally through both Ryk and Fz receptors. Finally, inhibiting both receptor types further reduced the proportion of responsive cells to $10 \%(1 / 10)$ (Fig. $5 E, J)$.

\section{Wnt5a-induced calcium activity involves IP3 receptors and TRP channels}

We next investigated the source of Wnt5a-evoked calcium activity. Calcium signaling involving release from intracellular stores is known to regulate axon growth and guidance (Takei et al., 1998; Ooashi et al., 2005; Jacques-Fricke et al., 2006). To determine whether this calcium signaling mechanism plays a role in effects of Wnt5a on cortical axons, we blocked IP3 receptors with 2-APB, which has been widely used as a blocker of IP3 receptors and store- 


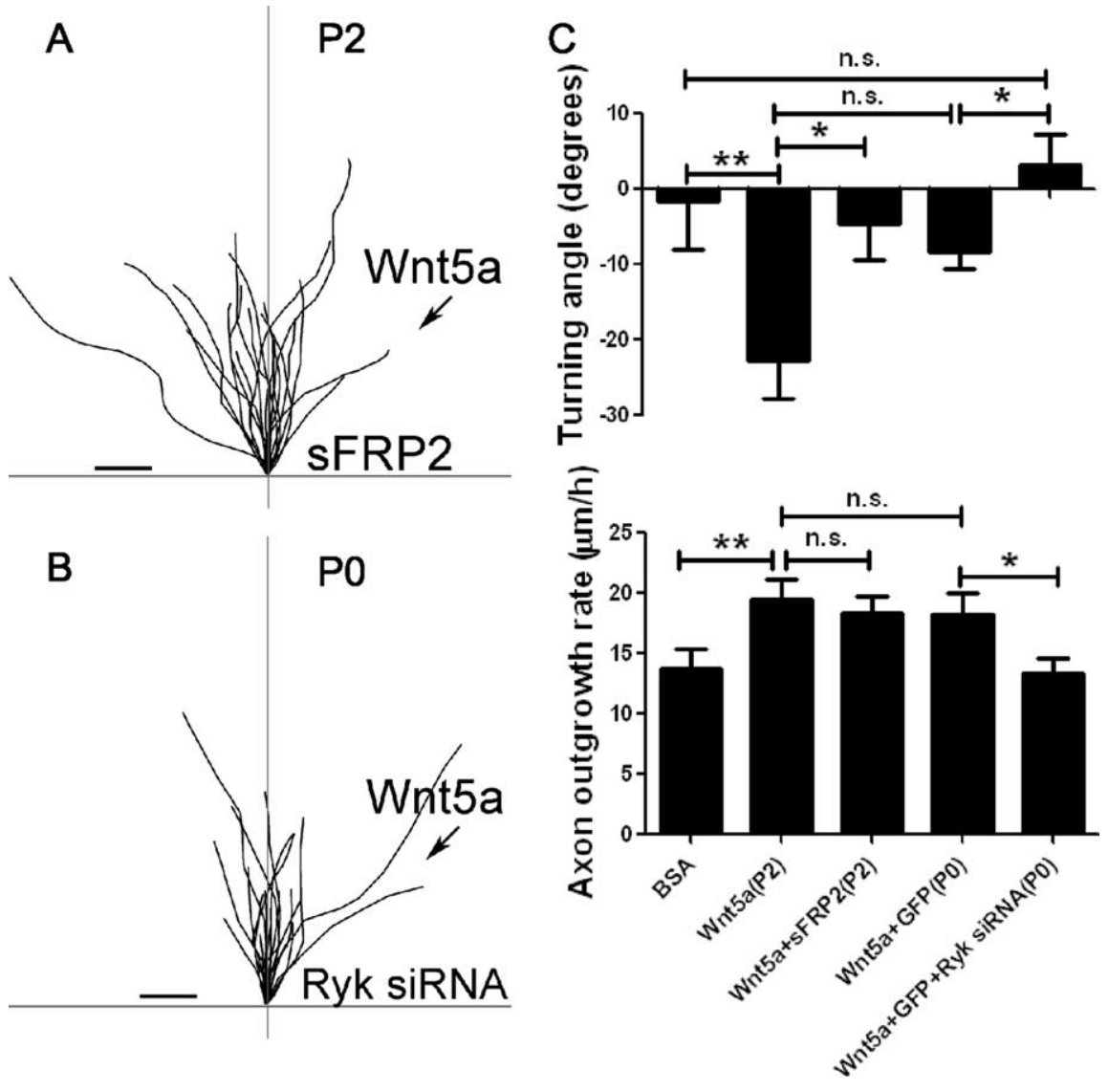

Figure 4. Fz receptors mediate Wnt5a-induced axon repulsion but not outgrowth, but Ryk receptors mediate both outgrowth and repulsion. $\boldsymbol{A}$, Tracings of trajectories of axons exposed to Wnt5a gradients in the presence of sFRP2 showing increased axon outgrowth but not repulsion. $\boldsymbol{B}$, Tracings of trajectories of $\mathrm{PO}$ axons exposed to Wnt5a gradients following Ryk knockdown, which prevents increased axon outgrowth as well as repulsion by Wnt5a. C, Bar graphs quantifying turning angles (top) and axon outgrowth rates (bottom) of axons in $\boldsymbol{A}$ and $\boldsymbol{B}$. Scale bar, $5 \mu \mathrm{m}$. In all histograms, ${ }^{*} p<0.05,{ }^{* *} p<0.01$; n.s., not significant.

operated channels (Bootman et al., 2002). In nine neurons showing calcium transients evoked by Wnt5a, 2-APB partially attenuated calcium activity in $5 / 9$ experiments in which the frequency was reduced from 10.4 to 3.5 transients/h (Fig. 5F). TRP channels have also been shown to be important for calcium signaling during growth cone guidance and axon outgrowth (Li et al., 2005; Shim et al., 2005; Wang and Poo, 2005). We therefore applied SKF96365, which is a general inhibitor of TRP channels (Wang and Poo, 2005). This treatment also partially attenuated calcium activity in $4 / 6$ experiments, reducing the frequency of calcium transients from 9.0 to 0.8 per hour after the addition of SKF96365 (Fig. 5G). When both blockers were applied together calcium activity evoked by Wnt5a was silenced (4/4 experiments) (Fig. 5H,I). Thus, Wnt5a-evoked calcium activity involves release from intracellular stores through IP3 receptors as well as calcium influx through TRP channels.

Wnt5a-induced axon outgrowth and repulsive guidance is mediated by distinct calcium signaling mechanisms

To determine the role of Wnt/calcium signaling in Wnt5a-induced axon outgrowth and guidance, we performed Wnt5a turning assays in the presence of $2 \mu \mathrm{M}$ BAPTA-AM, which chelates cytoplasmic calcium. As shown in Figure 6, $A$ and $B$, neurons treated with BAPTA fail to increase their rates of outgrowth or show repulsive turning in response to a Wnt5a gradient. Thus, as has been shown for other guidance cues such as netrin-1 and BDNF (Gomez and Zheng, 2006), calcium signaling is required for both of these Wnt-induced behaviors. Given our finding that Wnt5aevoked calcium activity involves calcium release from intracellular stores as well as calcium entry through TRP channels, we asked whether different modes of Wnt/calcium signaling could differentially mediate growth versus guidance of cortical axons as has been shown for several axon guidance cues (Ooashi et al., 2005; Jacques-Fricke et al., 2006). To address this question, we used $50 \mu \mathrm{M} 2$-APB or $3 \mu \mathrm{M}$ SKF96365 to block IP3 receptors and TRP channels, respectively. When a gradient of Wnt5a was applied to cortical axons from a pipette, application of 2-APB prevented increased outgrowth by Wnt5a but surprisingly did not prevent repulsive turning (Fig. $6 A, B$ ). Increased axon outgrowth was also blocked by 2-APB when Wnt5a was bath applied for $3 \mathrm{~d}$ (Fig. 6C). However, in the presence of SKF96365 both increased axon outgrowth and repulsive turning were inhibited in Wnt5a gradients (Fig. 6A, B). In longer-term 3 d experiments, application of SKF96365 also reduced axon outgrowth in the presence of bath-applied Wnt5a (Fig. 6D). These results suggest that the calcium signaling mechanisms underlying axon outgrowth are similar during shortterm $(1 \mathrm{~h})$ and long-term $(3 \mathrm{~d})$ exposure to Wnt5a. Together, these results show that Wnt5a-evoked calcium signaling is required for the growth-promoting and repulsive effects of Wnt5a and that distinct calcium signaling mechanisms are involved in each behavior. Thus, calcium release from stores through IP3 receptors mediates Wnt5aevoked axon outgrowth but not repulsive turning behaviors, whereas calcium influx through TRP channels mediates axon outgrowth as well as repulsive turning.

\section{Components of calcium signaling pathways are involved in Wnt5a-induced axon outgrowth}

To identify some of the upstream and downstream components of the calcium signaling pathways involved in Wnt5a-evoked axon outgrowth, we applied several pharmacological blockers and agonists to cortical neurons in the presence of Wnt5a and measured axon outgrowth over $3 \mathrm{~d}$ (supplemental Fig. 1, available at www.jneurosci.org as supplemental material). Phospholipase C (PLC), which is upstream of IP3, was blocked with $\mathrm{U}-73122$, a treatment that ultimately blocks calcium release through IP3 receptors. As expected, this prevented Wnt5a increases in axon outgrowth, confirming that this calcium signaling mechanism is required for axon outgrowth by Wnt5a. Conversely, to activate PLC, we applied m-3M3FBS and found that this treatment increased axon outgrowth, mimicking the effect of Wnt5a. To investigate the role of components downstream of calcium signaling, we applied 2 inhibitors to CaMKII, which has been shown to be important in axon outgrowth (Tang and Kalil, 2005). Application of either AIPII or KN62 alone had no effect on axon length but prevented increased axon outgrowth induced by Wnt5a. These results confirm that Wnt5a-induced axon outgrowth involves calcium signaling components previously associated with promotion of axon outgrowth. 
A

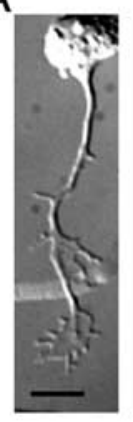

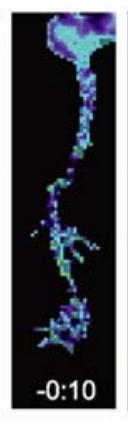

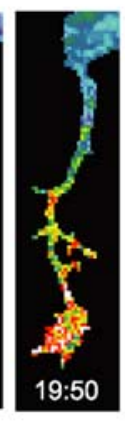

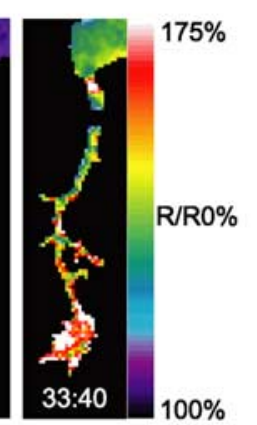

B

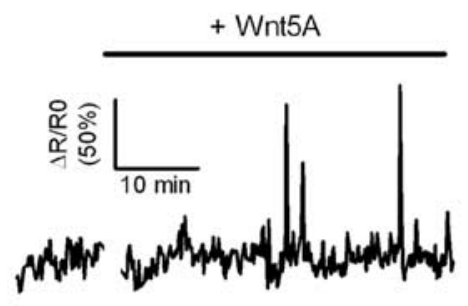

+ Wnt5A

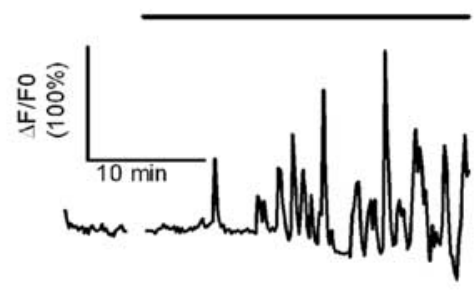

C

SFRP2 pretreated

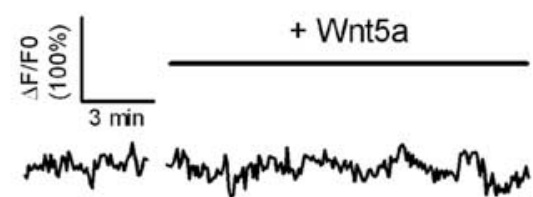

F

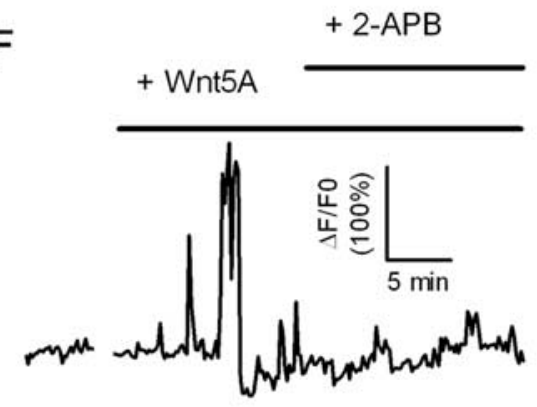

I

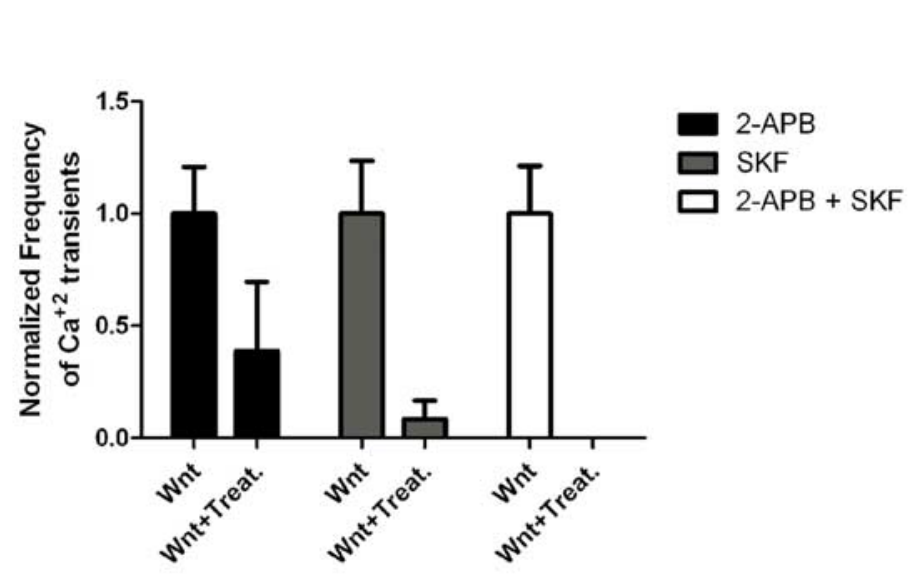

G

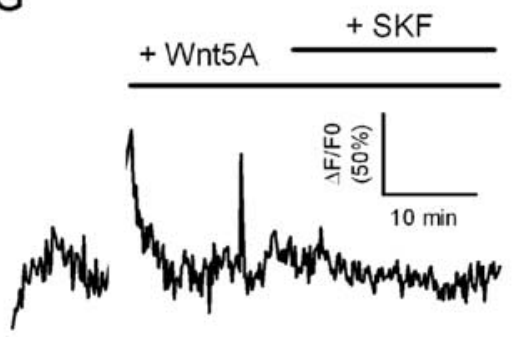

E

Ryk KD

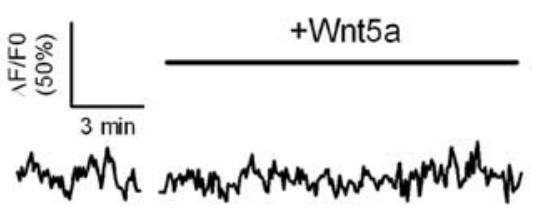

SFRP2 pretreated +Ryk KD

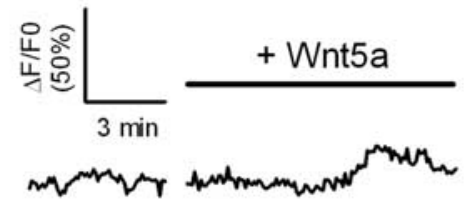

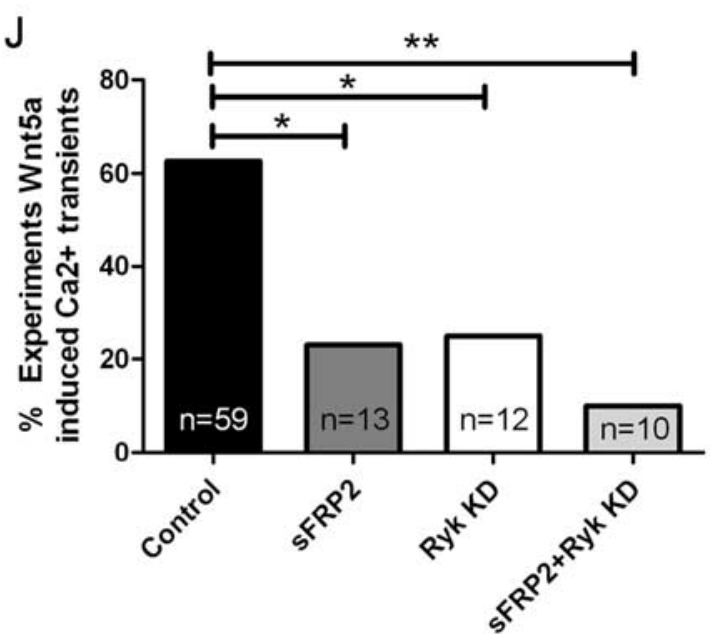

Pretreatment condition

Figure 5. Bath application of Wnt5a to cortical neurons evokes calcium activity through both Ryk and Fz receptors and blocking IP3 receptors or TRP channels attenuates this activity. A, DIC and matching ratiometric fluorescence time-lapse images of a cortical neuron, its axon and growth cone showing calcium activity before ( $-0: 10 \mathrm{~min})$ and after (19:50, 27:20,33:40 min) the addition of $400 \mathrm{ng} / \mathrm{ml} \mathrm{Wnt5a.} \mathrm{At} \mathrm{right,} \mathrm{the} \mathrm{tracings} \mathrm{show} \mathrm{increased} \mathrm{frequency} \mathrm{and} \mathrm{amplitude} \mathrm{of} \mathrm{calcium} \mathrm{transients} \mathrm{in} \mathrm{the} \mathrm{cortical} \mathrm{neuron} \mathrm{shown} \mathrm{at} \mathrm{left} \mathrm{beginning} 15$ min after addition of Wnt5a. $\boldsymbol{B}$, Additional example of Wnt5a-evoked calcium activity. ( $\boldsymbol{E}$, Tracing of calcium activity after application of Wnt5a in the presence of sFRP2 (200 ng/ml) (C) or $2 \mathrm{~d}$ after treatment with Ryk siRNA (D) or both (E). $\boldsymbol{F}$, Tracing of calcium activity evoked by Wnt5a before and after addition of 2-APB (50 $\mu \mathrm{M})$. G, Tracing of calcium activity evoked by Wnt5a before and after addition of SKF96365 (3 $\mu \mathrm{M})$. $\boldsymbol{H}$, Tracing of calcium activity evoked by Wnt5a before and after addition of both 2-APB and SKF96365.I, Quantification of frequencies of calcium transients evoked by Wnt5a before (bars labeled Wnt) and after the addition of calcium channel inhibitors (bars labeled Wnt + Treat.). Frequencies of calcium transients in neurons responsive to calcium channel inhibitors (2-APB, 5/9 neurons; SKF, 4/6 neurons; 2-APB plus SKF, 4/4 neurons) have been normalized to the baseline frequency of calcium transients evoked by Wnt5a before the addition of inhibitors. The absence of a bar in the 2-APB + SKF treatment group indicates that this treatment silenced calcium transients. J, Summary of experiments evoking calcium activity with Wnt5a after treatment with sFRP2 or Ryk siRNA $\left({ }^{* *} p<0.01\right.$, ${ }^{*} p<0.05$, Fisher's exact test). $n$, Number of axons in each condition. Scale bar, $10 \mu \mathrm{m}$. 

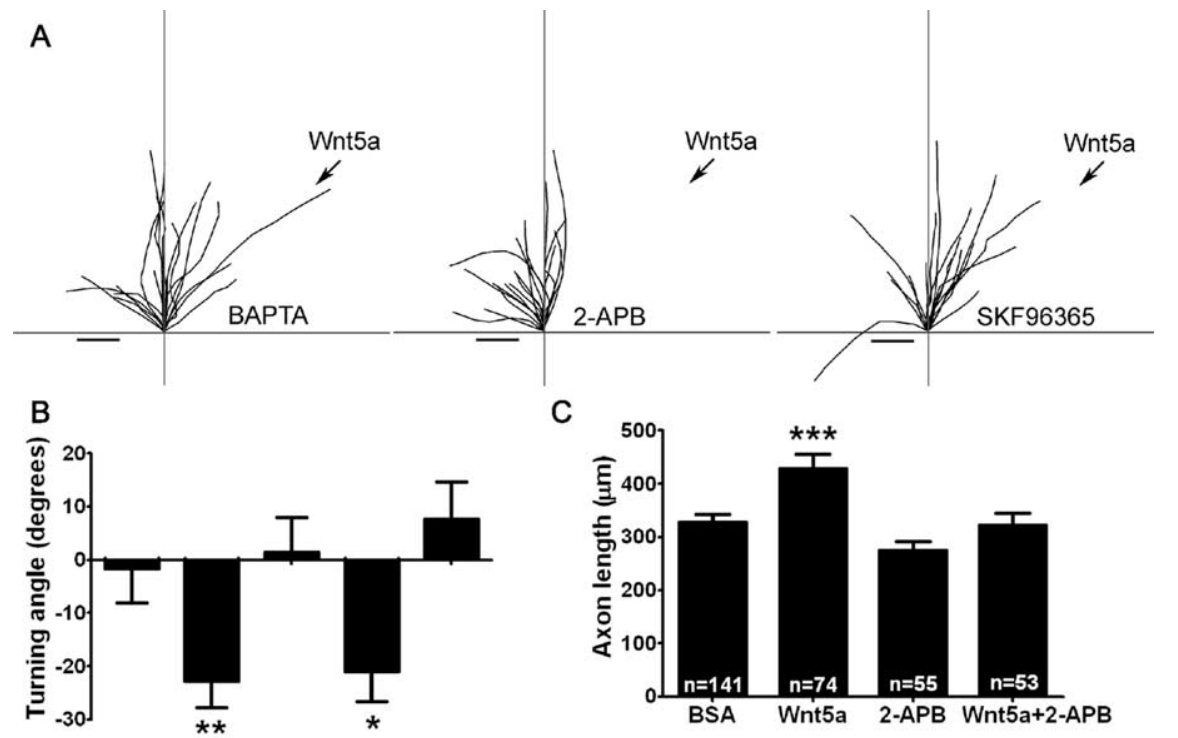

C
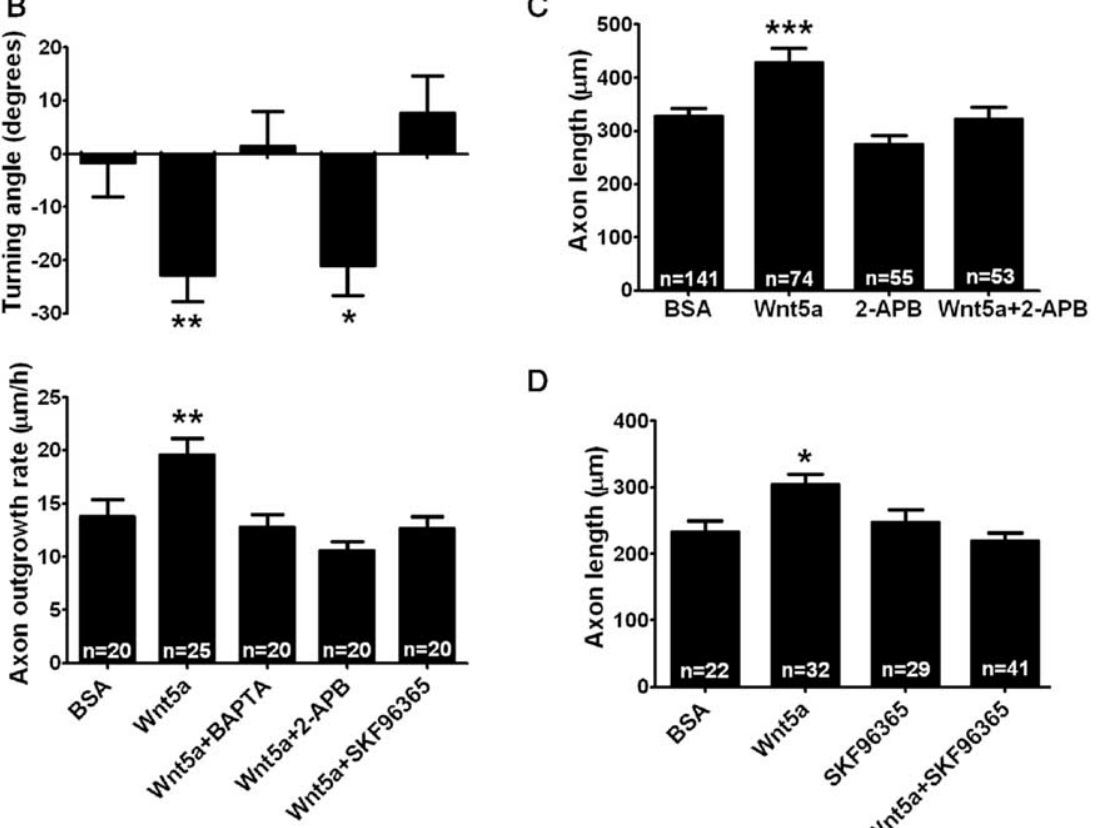

D

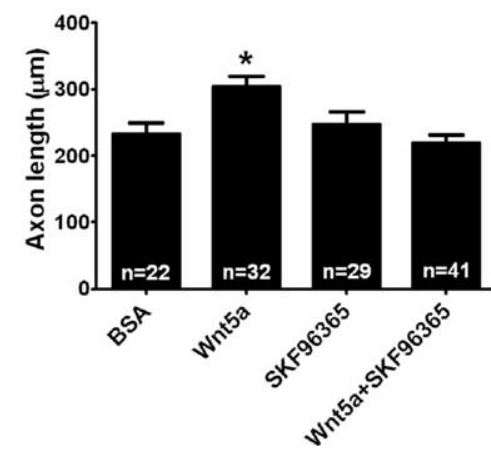

Figure 6. Calcium mediates Wnt5a-induced axon outgrowth and repulsive axon turning through distinct signaling mechanisms. $\boldsymbol{A}$, Tracings of trajectories of cortical axons in a Wnt5a turning assay in the presence of $2 \mu \mathrm{M}$ BAPTA-AM (a chelator of cytoplasmic calcium) (left), $50 \mu \mathrm{m}$ 2-APB (which blocks IP3 receptors) (middle), or $3 \mu \mathrm{m}$ SKF96365 (which blocks TRP channels) (right). Scale bar, $5 \mu \mathrm{m}$. $\boldsymbol{B}$, Bar graphs of turning angles (top) and rates of axon outgrowth (bottom) in conditions shown in $A$. In $\boldsymbol{B}$, the data for BSA and Wnt5a are the same as those shown in Figure 2D.C, D, Bar graphs of axon lengths in cultures treated for $3 \mathrm{~d}$ with bath-applied BSA, Wnt5a, 2-APB, SKF96365, or Wnt5a in the presence of 2-APB or SKF96365 from one experiment. Experiments were repeated three times. In all histograms, ${ }^{*} p<0.05,{ }^{* *} p<0.01$, and ${ }^{* * *} p<0.001$, and $n$, number of axons in each condition.

\section{Discussion}

Our results in dissociated cortical cultures reveal several novel effects of Wnt5a on axonal growth and guidance. These results are summarized in the model in Figure 7. First, while bath-applied Wnt5a promoted the outgrowth of cortical axons over several days, a point source of Wnt5a increased outgrowth rates of cortical axons within minutes while simultaneously inducing repulsive turning of their growth cones. Second, we show that activation of Ryk receptors is required to promote increased outgrowth of cortical axons by Wnt5a but that both Ryk and Fz receptors are necessary for Wnt5ainduced cortical axon repulsion. Third, we show for the first time that the Wnt/calcium signaling pathway, which is known to be mediated by Fz receptors, can also be activated by Ryk and is essential for axon outgrowth and repulsive turning. Interestingly, Wnt5apromoted axon outgrowth involves calcium release through IP3 receptors as well as calcium influx through TRP channels, whereas repulsive turning behaviors are mediated by TRP channels but do not require calcium release from stores through IP3 receptors. Thus, as shown in the schematic summary (Fig. 7), Wnt5a can simultaneously activate different receptors and different calcium signaling pathways at the growth cone and thereby elicit growth and guidance behaviors required for development of cortical axon pathways.

A puzzling aspect of Wnt5a signaling in developing corticospinal pathway is that function-blocking antibodies to Ryk introduced into the rostral spinal cord prevent the growth of corticospinal axons into the spinal cord and that axon outgrowth is inhibited from the side of cortical explants facing a source of secreted Wnt5a (Liu et al., 2005). Loss of Ryk in knock-out mice does not prevent the growth of callosal axons across the midline but impedes their escape from the midline into the contralateral hemisphere, as a result of aberrant axon trajectories (Keeble et al., 2006). These results suggest that in normal development cortical axons must navigate through a high-to-low Wnt5a gradient, which was demonstrated in both the spinal cord and corpus callosum. How can a chemorepellent Wnt5a gradient promote the growth of cortical axons through their efferent pathways? Our finding of simultaneous axon outgrowth and repulsive turning behaviors in response to Wnt5a gradients suggest a mechanism in which Wnt5a could repel corticospinal axons down the spinal cord (Zou and Lyuksyutova, 2007). This does not rule out the possibility that attractive guidance cues could counterbalance the repellent effects of Wnt5a (Bovolenta et al., 2006).

In this study, we found that in response to Wnt5a axon outgrowth is dependent on activation of Ryk receptors, whereas growth cone repulsion requires both Ryk and Fz. A previous study has shown that a source of secreted Wnt5a inhibits neurite outgrowth from explants of sensorimotor cortex. This inhibition was abolished by application of function-blocking Ryk antibodies (Liu et al., 2005), suggesting that Wnt5a acts via Ryk receptors to inhibit the extension of cortical axons from explants. In contrast, we found that Wnt5a promotes the growth of axons in dissociated cortical cultures and that increased axon outgrowth is prevented when Ryk receptors are blocked with function-blocking antibodies or the expression of Ryk is reduced by Ryk siRNA. The effect of Wnt5a on axon outgrowth of dissociated cortical neurons is consistent with a recent study showing that Wnt5a increases axon length and promotes axon differentiation of dissociated hippocampal neurons (Zhang et al., 2007). Ryk receptors have also been shown to mediate axon outgrowth of DRG neurons in response to Wnt3a (Lu et al., 2004). Previous studies have demonstrated the involvement of both Ryk and Fz receptors in axon guidance. Ryk, in response to Wnt5a, mediates repulsion of cortical axons (Liu et al., 2005; Keeble et al., 2006), which is consistent with our findings. However, a novel finding in our study is that Fz receptors also mediate cortical growth cone repulsion. In contrast, previous studies in the mammalian CNS have suggested that Fz receptors are involved primarily in growth cone attraction (Bovolenta et al., 2006). Since $10 \mathrm{Fz}$ receptors 
have been identified, it is possible that different members of the Fz family have different functions. For example in $\mathrm{Fz} 3$ knock-out mice cortical axon tracts, including the corpus callosum and corticospinal pathways, fail to develop, providing the first evidence that Fz signaling plays an important role in axon development (Wang et al., 2002). At present we do not know the identity of the Fz receptor(s) that mediate cortical growth cone repulsion and it is possible several $\mathrm{Fz}$ receptors are involved. Although sFRP is considered to block Wnt/Fz binding (Bafico et al., 1999; Liu et al., 2005; Bovolenta et al., 2006), it is not known whether sFRP effectively blocks all Fz receptors. Thus, we cannot completely eliminate the possibility that Fz receptors are involved in Wnt5apromoted axon outgrowth. Another possibility is that Ryk and Fz receptors interact, which has been shown biochemically by coimmunoprecipitation ( $\mathrm{Lu}$ et al., 2004). Although we cannot rule out this possibility, our finding that blocking Ryk receptors prevents Wnt5a-induced axon outgrowth but blocking $\mathrm{Fz}$ receptors has no effect on outgrowth suggests that at least in this context these receptors are able to function independently.

The noncanonical Wnt/calcium pathway is mediated by $\mathrm{Fz}$ receptors and has been shown to play a role in regulation of cell movement during gastrulation (Veeman et al., 2003a,b). Although nothing was known about the role of Wnt/calcium signaling in axon outgrowth and guidance, we considered it likely that such signaling pathways would also play a role in growth cone extension and turning behaviors (Zou, 2004). We found that calcium signaling is essential in transducing the effects of Wnt5a on cortical axon outgrowth and guidance and that this activity is mediated by Fz receptors. Since the noncanonical Wnt/calcium pathway is thought to involve downstream RhoGTPases (Veeman et al., 2003a), this Wnt signaling pathway could ultimately regulate growth cone behaviors by targeting cytoskeletal proteins (Gomez and Zheng, 2006). Interestingly, we also found that calcium signaling is mediated by Ryk, for which signal transduction mechanisms were previously unknown. This finding suggests that more than one class of Wnt receptors mediates calcium signaling. Although our results cannot link specific receptor types to distinct calcium signaling pathways, the finding that axon outgrowth involves Ryk but not Fz receptors suggests the possibility that different receptors may activate different signaling pathways.

Previous studies of calcium signaling pathways involved in axon outgrowth and growth cone turning in response to guidance cues demonstrated the importance of the mode of calcium entry in mediating different behaviors (Henley and Poo, 2004; Ooashi et al., 2005; Gomez and Zheng, 2006; Zheng and Poo, 2007). Axon outgrowth has been shown to involve calcium influx through L-type voltage-gated channels (Tang et al., 2003; Hutchins and Kalil, 2008). However, application of nifedipine to cortical neurons in the present study appeared to have no effect on Wnt-induced axon outgrowth (data not shown), suggesting that L-type channels were not involved. Previous studies have shown that IP3 receptors are involved in axon outgrowth (Takei

\section{Growth cone repulsion}

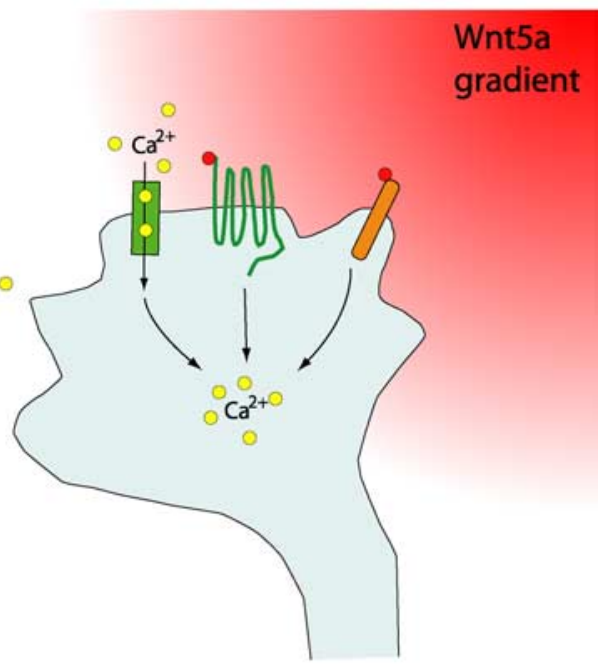

- IP3
W IP3 receptor

TRP

\section{Endoplasmic \\ Ryk receptor

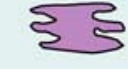 \\ reticulum}

Figure 7. Schematic summary showing Wnt receptors and calcium signaling components involved in cortical growth cone behaviors in response to Wnt5a. At left, increased cortical axon outgrowth is induced by Wnt5a, which involves activation of Ryk reticulum, and activation of PLC and CaMKII signaling. At right, growth cone repulsion by a Wnt5a gradient is mediated by both Ryk and Fz receptors and requires calcium entry through TRP channels. Symbols in the growth cone are shown in the box.

et al., 1998; Jacques-Fricke et al., 2006). Here we show that blocking IP3 receptors blocked the growth-promoting effects of Wnt5a. Moreover, pharmacologically inhibiting PLC, which is known to act upstream of IP3, eliminates the growth-promoting effects of Wnt5a, whereas a PLC agonist mimicked the effects of Wnt5a, confirming that 2-APB blocks the IP3 pathway. In addition we show for the first time that TRP channels are necessary for Wnt5a-induced axon outgrowth. Previous studies have shown that TRP channels are involved in axon outgrowth of Xenopus spinal neurons (Jacques-Fricke et al., 2006) and rat hippocampal neurons (Greka et al., 2003). However, in hippocampal neurons the use of dominant negative TRPC5 increased neurite outgrowth, suggesting that calcium entry through this channel normally functions to restrain the rate of axon outgrowth. One possibility is that promotion of cortical axon outgrowth occurs through calcium influx via a TRP channel other than TRPC5. Early postnatal rat cortical neurons have been shown to express TRPC4 as well as TRPC5 channels (Fowler et al., 2007).

We found that repulsive growth cone turning in response to Wnt5a also required activation of TRP channels. Consistent with our findings, several recent studies have shown the importance of TRP channels in growth cone turning behaviors in response to gradients of the guidance cues netrin-1 and BDNF (Li et al., 2005; Shim et al., 2005; Wang and Poo, 2005). In some cases (Li et al., 2005), activation of TRP channels required calcium release through IP3 receptors, an important component of axon guidance through TRP channels (Gomez and Zheng, 2006; Wen and Zheng, 2006). Surprisingly, we found that cortical growth cones in Wnt5a gradients can exhibit turning behaviors through TRP channel activation that did not require calcium release through IP3 receptors (Fig. 6). The fail- 
ure of OAG, an analog of DAG (diacylglycerol), to mimic the effects of Wnt5a on axon outgrowth and guidance (supplemental Fig. 2, available at www.jneurosci.org as supplemental material) suggests that TRP channels are not activated by DAG in response to Wnt5a. This is consistent with results in cerebellar granule cells (Li et al., 2005) responding to BDNF showing that DAG does not activate TRPC channels. At present it is not known how guidance cues such as Wnt5a activate growth cone TRP channels. Moreover, further studies will be needed to understand how the same guidance cue can evoke axon outgrowth and turning behaviors through different receptors and calcium signaling mechanisms.

\section{References}

Bafico A, Gazit A, Pramila T, Finch PW, Yaniv A, Aaronson SA (1999) Interaction of frizzled related protein (FRP) with Wnt ligands and the frizzled receptor suggests alternative mechanisms for FRP inhibition of Wnt signaling. J Biol Chem 274:16180-16187.

Bootman MD, Collins TJ, Mackenzie L, Roderick HL, Berridge MJ, Peppiatt CM (2002) 2-aminoethoxydiphenyl borate (2-APB) is a reliable blocker of store-operated Ca2 + entry but an inconsistent inhibitor of InsP3induced Ca2 + release. FASEB J 16:1145-1150.

Bovolenta P, Rodriguez J, Esteve P (2006) Frizzled/RYK mediated signalling in axon guidance. Development 133:4399-4408.

Ciani L, Salinas PC (2005) WNTs in the vertebrate nervous system: from patterning to neuronal connectivity. Nat Rev Neurosci 6:351-362.

Dent EW, Kalil K (2001) Axon branching requires interactions between dynamic microtubules and actin filaments. J Neurosci 21:9757-9769.

Dent EW, Kalil K (2003) Dynamic imaging of neuronal cytoskeleton. Methods Enzymol 361:390-407.

Dickson BJ (2002) Molecular mechanisms of axon guidance. Science 298:1959-1964.

Fowler MA, Sidiropoulou K, Ozkan ED, Phillips CW, Cooper DC (2007) Corticolimbic expression of TRPC4 and TRPC5 channels in the rodent brain. PLoS ONE 2:e573.

Gomez TM, Zheng JQ (2006) The molecular basis for calcium-dependent axon pathfinding. Nat Rev Neurosci 7:115-125.

Greka A, Navarro B, Oancea E, Duggan A, Clapham DE (2003) TRPC5 is a regulator of hippocampal neurite length and growth cone morphology. Nat Neurosci 6:837-845.

Halford MM, Oates AC, Hibbs ML, Wilks AF, Stacker SA (1999) Genomic structure and expression of the mouse growth factor receptor related to tyrosine kinases (Ryk). J Biol Chem 274:7379-7390.

Halford MM, Armes J, Buchert M, Meskenaite V, Grail D, Hibbs ML, Wilks AF, Farlie PG, Newgreen DF, Hovens CM, Stacker SA (2000) Rykdeficient mice exhibit craniofacial defects associated with perturbed Eph receptor crosstalk. Nat Genet 25:414-418.

Henley J, Poo MM (2004) Guiding neuronal growth cones using Ca2+ signals. Trends Cell Biol 14:320-330.

Hutchins BI, Kalil K (2008) Differential outgrowth of axons and their branches is regulated by localized calcium transients. J Neurosci 28:143-153.

Jacques-Fricke BT, Seow Y, Gottlieb PA, Sachs F, Gomez TM (2006) $\mathrm{Ca}^{2+}$ influx through mechanosensitive channels inhibits neurite outgrowth in opposition to other influx pathways and release from intracellular stores. J Neurosci 26:5656-5664.

Keeble TR, Halford MM, Seaman C, Kee N, Macheda M, Anderson RB, Stacker SA, Cooper HM (2006) The Wnt receptor Ryk is required for Wnt5a-mediated axon guidance on the contralateral side of the corpus callosum. J Neurosci 26:5840-5848.

Kohn AD, Moon RT (2005) Wnt and calcium signaling: beta-cateninindependent pathways. Cell Calcium 38:439-446.

Li Y, Jia YC, Cui K, Li N, Zheng ZY, Wang YZ, Yuan XB (2005) Essential role of TRPC channels in the guidance of nerve growth cones by brain-derived neurotrophic factor. Nature 434:894-898.

Liu Y, Shi J, Lu CC, Wang ZB, Lyuksyutova AI, Song XJ, Zou Y (2005) Ryk-mediated Wnt repulsion regulates posterior-directed growth of corticospinal tract. Nat Neurosci 8:1151-1159.

Logan CY, Nusse R (2004) The Wnt signaling pathway in development and disease. Annu Rev Cell Dev Biol 20:781-810.

Lohof AM, Quillan M, Dan Y, Poo MM (1992) Asymmetric modulation of cytosolic cAMP activity induces growth cone turning. J Neurosci 12:1253-1261.
Lu W, Yamamoto V, Ortega B, Baltimore D (2004) Mammalian Ryk is a Wnt coreceptor required for stimulation of neurite outgrowth. Cell 119:97-108.

Lyu J, Yamamoto V, Lu W (2008) Cleavage of the Wnt receptor Ryk regulates neuronal differentiation during cortical neurogenesis. Dev Cell 15:773-780.

Lyuksyutova AI, Lu CC, Milanesio N, King LA, Guo N, Wang Y, Nathans J, Tessier-Lavigne M, Zou Y (2003) Anterior-posterior guidance of commissural axons by Wnt-frizzled signaling. Science 302:1984-1988.

Nishiyama M, von Schimmelmann MJ, Togashi K, Findley WM, Hong K (2008) Membrane potential shifts caused by diffusible guidance signals direct growth-cone turning. Nat Neurosci 11:762-771.

Norris CR, Kalil K (1992) Development of callosal connections in the sensorimotor cortex of the hamster. J Comp Neurol 326:121-132.

Ooashi N, Futatsugi A, Yoshihara F, Mikoshiba K, Kamiguchi H (2005) Cell adhesion molecules regulate $\mathrm{Ca} 2+$-mediated steering of growth cones via cyclic AMP and ryanodine receptor type 3. J Cell Biol 170:1159-1167.

Reh T, Kalil K (1981) Development of the pyramidal tract in the hamster. I. A light microscopic study. J Comp Neurol 200:55-67.

Rodriguez J, Esteve P, Weinl C, Ruiz JM, Fermin Y, Trousse F, Dwivedy A, Holt C, Bovolenta P (2005) SFRP1 regulates the growth of retinal ganglion cell axons through the Fz2 receptor. Nat Neurosci 8:1301-1309.

Salinas PC, Zou Y (2008) Wnt signaling in neural circuit assembly. Annu Rev Neurosci 31:339-358.

Schmitt AM, Shi J, Wolf AM, Lu CC, King LA, Zou Y (2006) Wnt-Ryk signalling mediates medial-lateral retinotectal topographic mapping. Nature 439:31-37.

Shim S, Goh EL, Ge S, Sailor K, Yuan JP, Roderick HL, Bootman MD, Worley PF, Song H, Ming GL (2005) XTRPC1-dependent chemotropic guidance of neuronal growth cones. Nat Neurosci 8:730-735.

Shimogori T, VanSant J, Paik E, Grove EA (2004) Members of the Wnt, Fz, and Frp gene families expressed in postnatal mouse cerebral cortex. J Comp Neurol 473:496-510.

Slusarski DC, Corces VG, Moon RT (1997) Interaction of Wnt and a Frizzled homologue triggers G-protein-linked phosphatidylinositol signalling. Nature 390:410-413.

Takei K, Shin RM, Inoue T, Kato K, Mikoshiba K (1998) Regulation of nerve growth mediated by inositol 1,4,5-trisphosphate receptors in growth cones. Science 282:1705-1708.

Tang F, Kalil K (2005) Netrin-1 induces axon branching in developing cortical neurons by frequency-dependent calcium signaling pathways. J Neurosci 25:6702-6715.

Tang F, Dent EW, Kalil K (2003) Spontaneous calcium transients in developing cortical neurons regulate axon outgrowth. J Neurosci 23:927-936.

Trivier E, Ganesan TS (2002) RYK, a catalytically inactive receptor tyrosine kinase, associates with EphB2 and EphB3 but does not interact with AF-6. J Biol Chem 277:23037-23043.

Veeman MT, Axelrod JD, Moon RT (2003a) A second canon. Functions and mechanisms of beta-catenin-independent Wnt signaling. Dev Cell 5:367-377.

Veeman MT, Slusarski DC, Kaykas A, Louie SH, Moon RT (2003b) Zebrafish prickle, a modulator of noncanonical Wnt/Fz signaling, regulates gastrulation movements. Curr Biol 13:680-685.

Wang GX, Poo MM (2005) Requirement of TRPC channels in netrin-1induced chemotropic turning of nerve growth cones. Nature 434:898-904.

Wang Y, Thekdi N, Smallwood PM, Macke JP, Nathans J (2002) Frizzled-3 is required for the development of major fiber tracts in the rostral CNS. J Neurosci 22:8563-8573.

Wen Z, Zheng JQ (2006) Directional guidance of nerve growth cones. Curr Opin Neurobiol 16:52-58.

Yoshikawa S, McKinnon RD, Kokel M, Thomas JB (2003) Wnt-mediated axon guidance via the Drosophila Derailed receptor. Nature 422:583-588.

Zhang X, Zhu J, Yang GY, Wang QJ, Qian L, Chen YM, Chen F, Tao Y, Hu HS, Wang T, Luo ZG (2007) Dishevelled promotes axon differentiation by regulating atypical protein kinase C. Nat Cell Biol 9:743-754.

Zheng JQ, Poo MM (2007) Calcium signaling in neuronal motility. Annu Rev Cell Dev Biol 23:375-404.

Zheng JQ, Felder M, Connor JA, Poo MM (1994) Turning of nerve growth cones induced by neurotransmitters. Nature 368:140-144.

Zou Y (2004) Wnt signaling in axon guidance. Trends Neurosci 27:528-532.

Zou Y, Lyuksyutova AI (2007) Morphogens as conserved axon guidance cues. Curr Opin Neurobiol 17:22-28. 